Copyright (C) 2013 IEEE. Personal use of this material is permitted. Permission from IEEE must be obtained for all other uses, in any current or future media, including reprinting/republishing this material for advertising or promotional purposes, creating new collective works, for resale or redistribution to servers or lists, or reuse of any copyrighted component of this work in other works. 


\section{Channel Estimation Based on Compressed Sensing in High-speed Underwater Acoustic Communication}

\author{
Sichuan Guo, Zhiqiang He, Weipeng Jiang, \\ Yuanbiao $\mathrm{Ou}$, and Kai Niu \\ Key Lab of Universal Wireless Communications, \\ Ministry of Education \\ Beijing University of Posts and Telecommunications \\ Beijing, China
}

\author{
Yue Rong*, Michael Caley ${ }^{\dagger}$, \\ and Alec J Duncan ${ }^{\dagger}$ \\ *Department of Electrical and Computer Engineering \\ ${ }^{\dagger}$ Department of Imaging and Applied Physics \\ Curtin University, Bentley, WA, Australia
}

\begin{abstract}
The underwater acoustic (UA) channel is dispersive in both time and frequency with severe frequency-dependent signal attenuation. Efficient channel estimation and tracking are crucial to coherent high-rate UA communication. In this paper, we propose a new compressed sensing (CS) based channel estimation method with block-by-block channel tracking for UA communication. Compared with conventional channel estimation algorithms, the proposed method efficiently exploits the sparsity of the UA channel, and improves the channel tracking capability of UA communication system. The proposed algorithm was tested during our UA communication experiment conducted in December 2012 in the Indian Ocean off Rottnest Island, Western Australia. At a data rate of $8 \mathrm{kbps}$ (QPSK constellations), average uncoded bit-error-rates (BERs) of 3\% and $14 \%$ have been achieved over $1 \mathrm{~km}$ and $6 \mathrm{~km}$ ranges, respectively, using MMSE equalization based on the proposed channel estimation and tracking method.
\end{abstract}

Index Terms-Channel estimation, channel tracking, compressed sensing, underwater acoustic channel.

\section{INTRODUCTION}

The underwater acoustic (UA) channel is affected by many factors such as temperature, salinity, depth, storms, ocean currents, and interface reflections. In underwater communications, due to the effects of wind and currents, the relative motion between a drifting transmitter and receiver can reach a few meters per second even under mild weather conditions. Considering that the speed of sound in water is around 1500 $\mathrm{m} / \mathrm{s}$, such motion generates strong Doppler effects. On the other hand, the bandwidth available for UA communication is limited and is inversely proportional to the transmission range [1]. It is shown in [2] that the range-rate product of most UA communication systems is upper-bounded by 40 $\mathrm{km}-\mathrm{kbps}$. This indicates that the bandwidth spent for channel training should be limited in order to achieve high-rate UA communication. A highly varying environment and limited bandwidth make efficient channel estimation and tracking in UA communication extremely challenging.

It has been shown in [3] that UA channels are sparse, with only a few propagation paths carrying the significant portion of channel energy. The sparsity of UA channels can be exploited

This work was supported by the National Science and Technology Major Project of China (No. 2012ZX03004005-002) and the Australian Research Council's Discovery Projects funding scheme (Project Number DP110100736) to improve the accuracy of channel estimation, for example, by the compressed sensing (CS) technique [4], [5]. In particular, sparse channel estimation can be implemented through a greedy method named matching pursuit (MP) algorithm [6][8]. When comparing the MP method [6] and the orthogonal MP (OMP) method [7], the latter one has a faster convergence speed and a better estimation by avoiding the so-called reelection phenomenon. On the other hand, the CoSaMP method [8] has the dual advantages of greedy algorithm and convex method, and performs better on both reconstruction and computing. Therefore, OMP and CoSaMP methods are both used as CS-based channel estimation algorithms in our paper.

Both the OMP and CoSaMP algorithms only work well in quasi-static channels. To have accurate channel estimation in the fast time-varying UA channel, the CS-based channel training should be performed periodically. The channel between two adjacent training sequences can be treated as quasi-static if the channel training frequency is sufficiently high. However, channel training sequences consume precious bandwidth in UA communications.

To increase the bandwidth efficiency of channel estimation, a block-by-block channel tracking method is developed in [9], where the time-varying channel is tracked by a decisiondirected time-domain least-squares (TD-LS) algorithm within each data block using the data estimated in that block. In this way, the UA channel can be estimated and tracked with a much shorter training sequence. Moreover, the computational complexity of the block-wise channel tracking method is much smaller compared with symbol-by-symbol channel tracking methods. However, the LS algorithm used in [9] is not the best choice for the UA channel, since it does not exploit the sparsity of the channel.

In this paper, we propose a new algorithm that combines the advantages of CS-based channel estimation and block-byblock channel tracking for UA communication. The proposed UA channel estimation algorithm is tested in our UA communication experiment conducted in December 2012 in the Indian Ocean off Rottnest Island, Western Australia. At a data rate of $8 \mathrm{kbps}$ with QPSK constellations, average uncoded biterror-rates of $3 \%$ and $14 \%$ have been achieved over $1 \mathrm{~km}$ and $6 \mathrm{~km}$ ranges, respectively, using the MMSE equalization based 


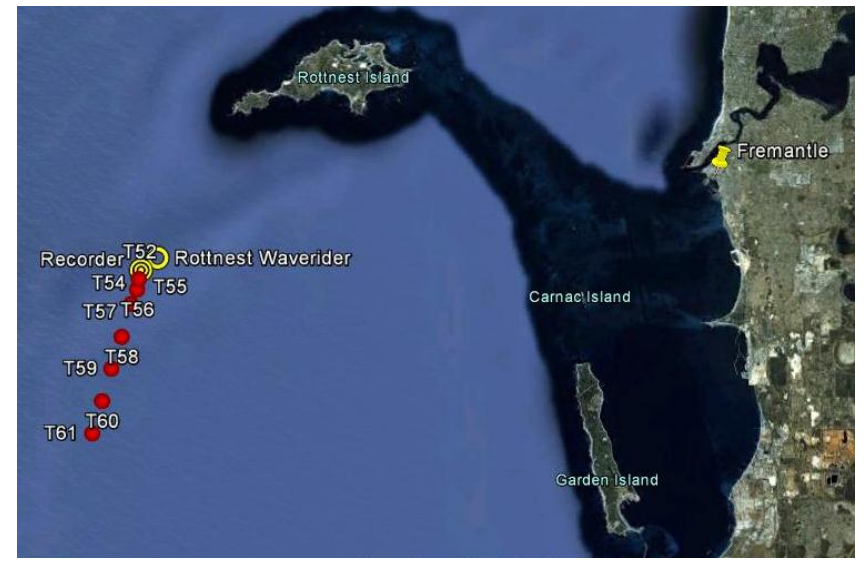

Fig. 1. General location of the experiment environment along 50m depth contour

on the proposed channel estimation and tracking method.

The rest of this paper is organized as follows. In Section II, our UA communication experiment is introduced. The system model for channel estimation and tracking is given in Section III. The OMP and CoSaMP based channel estimation algorithms are developed in Section IV. The results obtained using the proposed algorithm are presented in Section V, and conclusions are drawn in Section IV. We use the following notations throughout the paper: column vectors and matrices are denoted by lower case and upper case bold face symbols, respectively. The Moore-Penrose matrix pseudo inverse is denoted as $\mathbf{X}^{\dagger}$.

\section{EXPERIMENT ARRANGEMENT}

An UA communication experiment was conducted in December 2012 over distances of $125 \mathrm{~m}$ to $10 \mathrm{~km}$ in the Indian Ocean off Rottnest Island, Western Australia, as shown in Figure 1. The receiver (recorder) was located on the sea bed close to the Rottnest Waverider Buoy. The red dots with labels of T52, T54, T55, T56, T57, T58, T59, T60, and T61 denote the transmitter positions which were $125 \mathrm{~m}, 250 \mathrm{~m}, 500 \mathrm{~m}, 1$ $\mathrm{km}, 2 \mathrm{~km}, 4 \mathrm{~km}, 6 \mathrm{~km}, 8 \mathrm{~km}$, and $10 \mathrm{~km}$ from the receiver, respectively. The average water depth was $50 \mathrm{~m}$.

The transmitter and receiver arrangements are illustrated in Figure 2. At the transmitter, a single transducer was attached to a drifting vessel through cable, and the nominal transducer depth was $20 \mathrm{~m}$. A single hydrophone at the receiver was attached through a cable at $1 \mathrm{~m}$ above the seabed. A drifting transmitter was selected to give flexibility in exploring different communication ranges, however this arrangement also allows movement of the transducer and hydrophone, increasing the Doppler shifts and Doppler spreading, and making channel estimation and tracking more challenging. According to the GPS data, at the $1 \mathrm{~km}$ range, the average drift speed of the vessel was $0.96 \mathrm{~m} / \mathrm{s}$, with the peak drift speed of $1.7 \mathrm{~m} / \mathrm{s}$.

Signals were transmitted at the $12 \mathrm{kHz}$ centre frequency of the transmit transducer. The system bandwidth was 4 kHz. Transmitted and received signals were sampled with

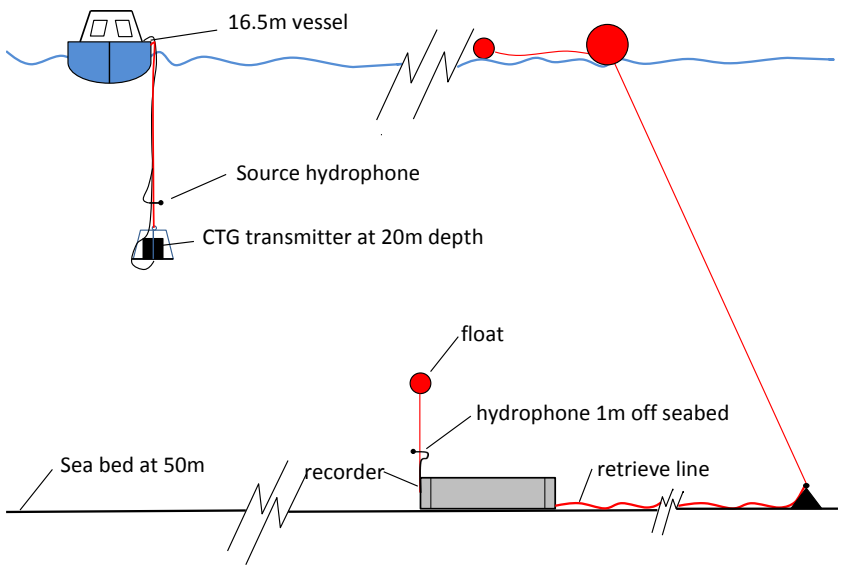

Fig. 2. Transmitter and receiver diagram

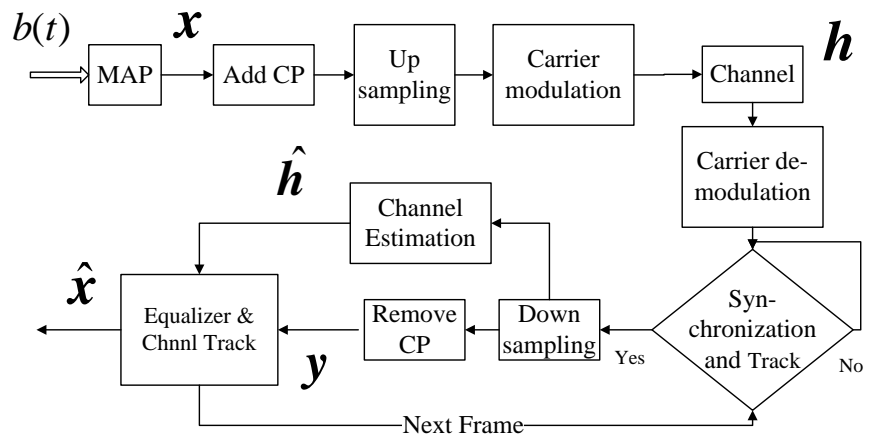

Fig. 3. System model

24 bit resolution at $96 \mathrm{kHz}$. 8PSK and QPSK symbols were transmitted at ranges of $125 \mathrm{~m}, 250 \mathrm{~m}, 500 \mathrm{~m}, 1 \mathrm{~km}, 2 \mathrm{~km}$, and $4 \mathrm{~km}$. QPSK and BPSK symbols were transmitted at the ranges of $6 \mathrm{~km}$ and $8 \mathrm{~km}$. At a range of $10 \mathrm{~km}$, BPSK symbols were transmitted.

\section{SYSTEM MODEL}

The block diagram of our UA communication system is shown in Figure 3.

At the channel training stage, the system input-output relationship is given by

$\left[\begin{array}{l}y(1) \\ y(2) \\ \vdots \\ y(M)\end{array}\right]=\left[\begin{array}{cccc}x(1) & x(0) & \cdots & x(2-N) \\ x(2) & x(1) & \cdots & x(3-N) \\ \vdots & \vdots & \ddots & \vdots \\ x(M) & x(M-1) & \cdots & x(M+1-N)\end{array}\right]\left[\begin{array}{l}h(1) \\ h(2) \\ \vdots \\ h(N)\end{array}\right]+\left[\begin{array}{l}v(1) \\ v(2) \\ \vdots \\ v(N)\end{array}\right]$

where $x(i), y(i), v(i)$ are the training signal, the received signal, and the additive noise, respectively, $h(i)$ denotes the impulse response of the UA channel, $N$ is the length of the channel and $M$ represents the length of received signal that is observed. Equation (1) can be rewritten using matrix-vector notation as

$$
\boldsymbol{y}=\mathbf{X} \boldsymbol{h}+\boldsymbol{v}
$$




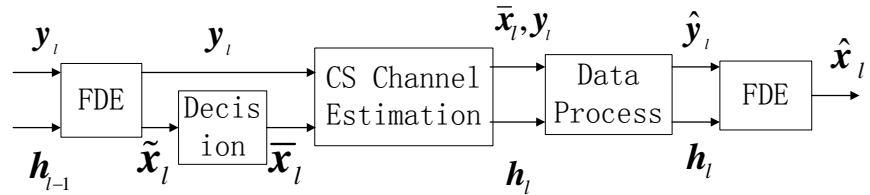

Fig. 4. Block diagram of the proposed channel estimation tracking method

where $\mathbf{X}$ is the training matrix. The problem we face is how to obtain the channel information $\boldsymbol{h}$ given the matrix $\mathbf{X}$ and the received signal $\boldsymbol{y}$.

It can be seen from (2) that the length of the training sequence should be larger than the channel memory length. In high-rate UA communications, the channel memory length is often on the order of a hundred symbols. On the other hand, due to the severe Doppler effect, the coherence time of the UA channel only spans about a couple of hundred symbols. This makes accurate UA channel estimation very challenging without compromising the system bandwidth efficiency. To address this challenge, in this paper, we propose a decisiondirected CS-based method to estimate and track the channel impulse response of fast-varying UA channels in a block-byblock manner as shown in Figure 4.

The initial channel estimation is obtained by the fixedlength training sequence. Then the detected symbols within each block are used to re-estimate the current channel which can be employed to correct the current data block and then equalize the next received data block. Since the length of data blocks is sufficiently short, we can assume that the channel is static within each block. Assuming that one data frame is divided into $L$ blocks, the specific steps are shown below.

1) Based on the known training sequence, an initial channel estimation $\boldsymbol{h}_{0}$ is obtained using the CS-based algorithm.

2) Then we track the channel of the $l$-th block $\boldsymbol{h}_{l}$, where $l=1,2, \ldots, L$, through the following steps.

a) Using the channel estimation of the previous block $\boldsymbol{h}_{l-1}$, we obtain a rough estimation $\tilde{\boldsymbol{x}}_{l}$ of the transmit signals by using the MMSE-based overlapped-window frequency-domain equalization (FDE) method. The cyclic prefix (CP) is only added ahead of each frame and there is no cyclic prefix in the data block. Therefore, the accuracy of $\tilde{\boldsymbol{x}}_{l}$ is not very high.

b) After the decision of $\tilde{\boldsymbol{x}}_{l}$ has been made to the corresponding constellation points, $\overline{\boldsymbol{x}}_{l}$ is obtained. Then the current channel estimation $\boldsymbol{h}_{l}$ can be obtained using known $\overline{\boldsymbol{x}}_{l}$ through CS-based method.

c) With the information of the estimated current channel, we can process the received signal $\boldsymbol{y}_{l}$ to obtain $\hat{\boldsymbol{y}}_{l}$, which is suitable for the FDE method.

d) Estimate the transmit signal $\hat{\boldsymbol{x}}_{l}$ of the current block with a second MMSE FDE process using $\hat{\boldsymbol{y}}_{l}$ and $\boldsymbol{h}_{l}$.

\section{Compressed Sensing Methods}

It can be seen from (1) that $\mathbf{X}$ is an $M \times N$ Toeplitz matrix of known training sequence. The impulse response of the UA channel $\boldsymbol{h}$ is the sparse signal that we want to reconstruct using $\mathbf{X}$ and the received signal $\boldsymbol{y}$. The CS theory can be applied to estimate $\boldsymbol{h}$. In the terminology of the CS theory, $\|\boldsymbol{h}\|_{0}=k$ is the sparsity of the channel, $\mathbf{X}$ is known as a dictionary, whose column vectors $\boldsymbol{x}_{i}(i=1,2, \ldots, N)$ are called atoms.

One of the fundamental problems in CS is to identify the measure matrices that are sufficient to ensure precise reconstruction of sparse vector $\boldsymbol{h}$ from observation vector $\boldsymbol{y}$. Matrices which have this character are named as CS matrices. It has been proven in [10] that an $M \times N$ Toeplitz matrix $\mathbf{X}$ of the following form is a CS matrix with high probability.

$$
\mathbf{X}=\left[\begin{array}{cccc}
x(1) & x(0) & \cdots & x(2-N) \\
x(2) & x(1) & \cdots & x(3-N) \\
\vdots & \vdots & \ddots & \vdots \\
x(M) & x(M-1) & \cdots & x(M+1-N)
\end{array}\right]
$$

This matrix is used as the measure matrix of channel tracking within the data blocks in our UA communication experiment. As an extension of the Toeplitz matrix (3), a circulant matrix given by (4) below has been proven a CS matrix with high probability. Circulant matrix (4) is the measure matrix of initial channel estimation within each frame and Toeplitz matrix (3) is the measure matrix of the following channel estimation when a FDE is used.

$$
\mathbf{X}=\left[\begin{array}{cccc}
x(1) & x(M) & \cdots & x(M-N+2) \\
x(2) & x(1) & \cdots & x(M-N+3) \\
\vdots & \vdots & \ddots & \vdots \\
x(M) & x(M-1) & \cdots & x(M-N+1)
\end{array}\right]
$$

After the recoverability of the measure matrix has been proven, in the following, we introduce two CS methods that are used in this paper.

\section{A. The OMP method}

The main idea of the OMP method is explained below. Since $\boldsymbol{h}$ has only $k$ nonzero elements, the received signal vector $\boldsymbol{y}=\mathbf{X} \boldsymbol{h}+\boldsymbol{v}$ is a linear combination of $k$ columns from $\mathbf{X}$ when the noise vector $\boldsymbol{v}$ is ignored. In the terminology of sparse approximation, we say that $\boldsymbol{y}$ has a $k$-term representation over the dictionary $\mathbf{X}$. To identify the UA channel impulse response $\boldsymbol{h}$, we need to determine the columns of $\mathbf{X}$ that are spanned by $\boldsymbol{y}$. In the OMP method, these columns are determined in an iterative fashion. At each iteration, we choose the column of $\mathbf{X}$ that is most strongly correlated with the remaining part of $\boldsymbol{y}$. Then we subtract off its contribution from $\boldsymbol{y}$. This procedure continues until all columns are identified, or the residual is sufficiently small. Then the estimated UA channel $\hat{\boldsymbol{h}}$ is obtained.

As the OMP method ensures orthogonality among atoms selected throughout iterations, it reduces the number of iterations significantly. However, the OMP method takes only 
one atom in each iteration, which is inefficient in term of the computational complexity, especially for $\boldsymbol{h}$ with a large dimension. This makes the OMP method a suboptimal choice in a high-rate UA system. In the next subsection, we introduce a faster CS method called CoSaMP.

\section{B. The CoSaMP method}

CoSaMP is a signal reconstruction method proposed in 2009 [10]. It has the stability of the basis pursuit (BP) algorithm as well as the quick and easy implementation of the greedy algorithm such as OMP, which are important to the reliability and effectiveness of the UA channel estimation. The specific steps of this algorithm are as follows.

1) Initialization: Set the sparsity of the UA channel as $k$. Assume that $\hat{\boldsymbol{h}}_{0}$ is an $N$-dimension zero vector. Set the residual as $\boldsymbol{r}_{0}=\boldsymbol{y}$ and the number of iterations as $l=1$.

2) The steps for the $l$-th iteration $(l=1,2, \ldots)$ are given below.

a) Compute the correlation $\left|\left\langle\boldsymbol{r}_{l-1}, \boldsymbol{x}_{i}\right\rangle\right|$ between the residual $\boldsymbol{r}_{l-1}$ and the columns of $\mathbf{X}$ and choose $2 k$ columns that have the largest value. Denote $\boldsymbol{g}_{l}$ as the indexes of the $2 k$ columns selected from $\mathbf{X}$.

b) Combine the set of index $\boldsymbol{t}_{l}=\boldsymbol{g}_{l} \cup$ $\sup \left\{\hat{\boldsymbol{h}}_{l-1}\right\}$, where $\sup \left\{\hat{\boldsymbol{h}}_{l-1}\right\}$ represents the indexes of nonzero values in $\hat{\boldsymbol{h}}_{l-1}$.

c) Obtain the estimated vector $\boldsymbol{b}_{l}$ by the LS algorithm. The elements of $\boldsymbol{b}_{l}$ with index $\boldsymbol{t}_{l}$ are given by $\mathbf{X}_{\boldsymbol{t}}^{\dagger} \boldsymbol{y}$, and other elements of $\boldsymbol{b}_{l}$ are set as zero. $\mathbf{X}_{\boldsymbol{t}_{l}}$ is a matrix consisting of the columns of $\mathbf{X}$ with index $\boldsymbol{t}_{l}$.

d) Obtain the new estimation $\hat{\boldsymbol{h}}_{l}$ as the largest $k$ elements of $\boldsymbol{b}_{l}$.

e) Update the residual: $\boldsymbol{r}_{l}=\boldsymbol{y}-\mathbf{X} \hat{\boldsymbol{h}}_{l}$.

The UA channel estimation $\hat{\boldsymbol{h}}$ is obtained after $k / 2$ iterations or when the residual is sufficiently small.

\section{Test Results From The Sea Experiment}

In this section, we present the performance of the proposed channel estimation algorithm in the UA communication experiment conducted in December 2012 in the Indian Ocean off the Rottnest Island, Western Australian.

The typical time-varying equivalent channel impulse responses (CIRs) during one data block are shown in Figure 5 and Figure 6 for communications over $1 \mathrm{~km}$ and $6 \mathrm{~km}$ range, respectively. These figures show the channels estimated by the training sequence and the 4 th data block detected. They have all been normalized by the maximum of CIR in $1 \mathrm{~km}$ system, which is estimated by training sequence. It can be seen that due to smaller channel attenuation in shorter range communication, the amplitude of CIRs of the $1 \mathrm{~km}$ system are larger than those of the $6 \mathrm{~km}$ system. It can also be observed from these two figures that the amplitude of CIRs are varying very fast even in one data frame.

Figure 7 shows the average uncoded BER with QPSK constellation at different range. The system data rate is 8

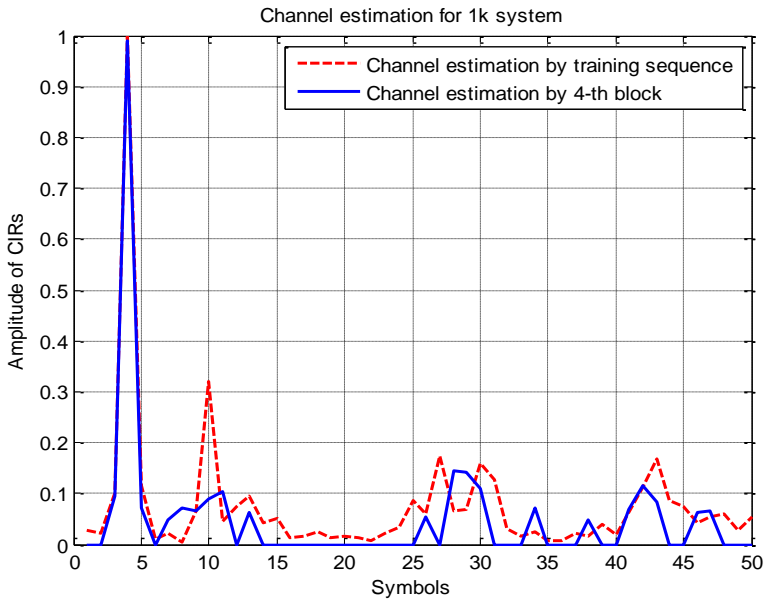

Fig. 5. The CIRs in one frame duration for the $1 \mathrm{~km}$ system

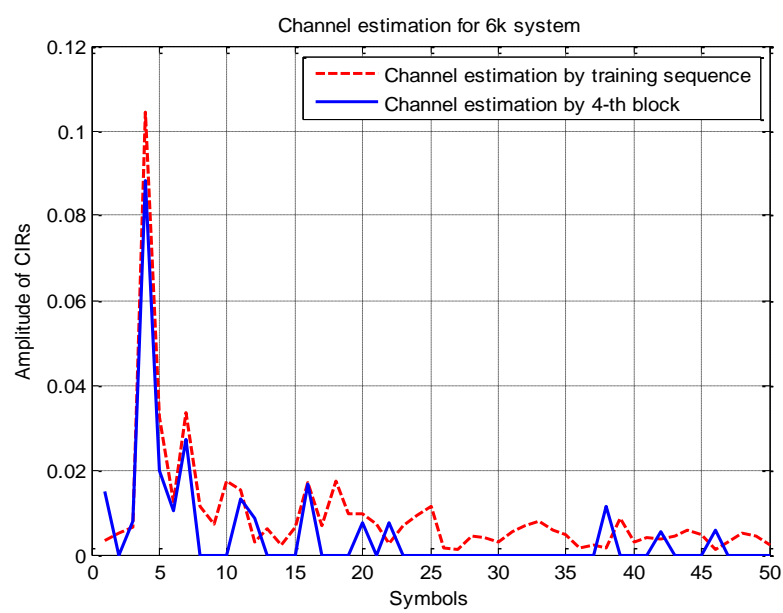

Fig. 6. The CIRs in one frame duration for the $6 \mathrm{~km}$ system

kbps. We compare the proposed CS-based channel estimation method with block-by-block channel tracking with the CSbased channel estimation without channel tracking. The OMP algorithm is used for both methods. It can be clearly seen from Figure 7 that our block-by-block channel estimation and tracking method with CS-based channel estimation performs better than the traditional CS-based channel estimation, and therefore, provides more accurate channel estimation for the channel equalization.

Figure 8 shows the performance comparison of the traditional LS channel estimation method and the channel estimation algorithms based on CS in terms of the average uncoded BER versus the communication range. The system data rate is $8 \mathrm{kbps}$. The OMP and CoSaMP methods are adopted to implement the CS-based channel estimation. All methods are tested in the communication ranges of $1 \mathrm{~km}, 2 \mathrm{~km}, 4 \mathrm{~km}$, $6 \mathrm{~km}$, and $8 \mathrm{~km}$, respectively in our experiment. All three methods adopt the block-by-block channel tracking approach.

It can be seen from Figure 8 that the channel estimation 


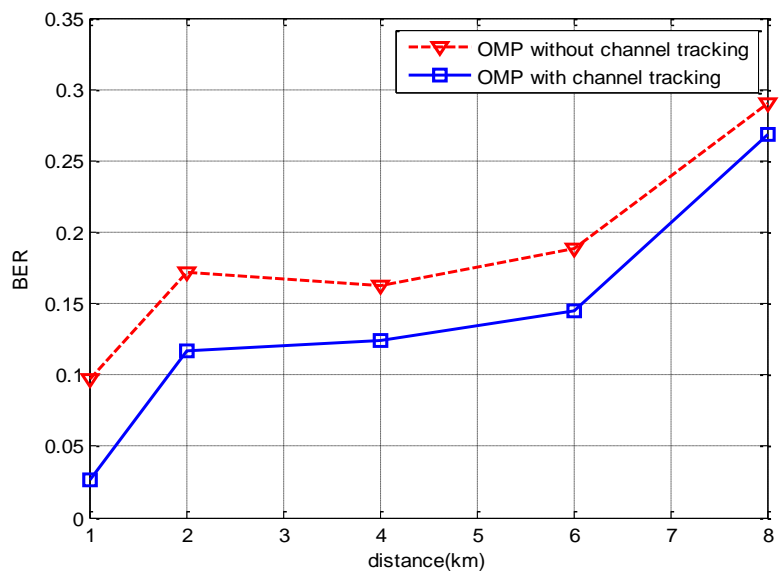

Fig. 7. BER performance of different channel estimation methods

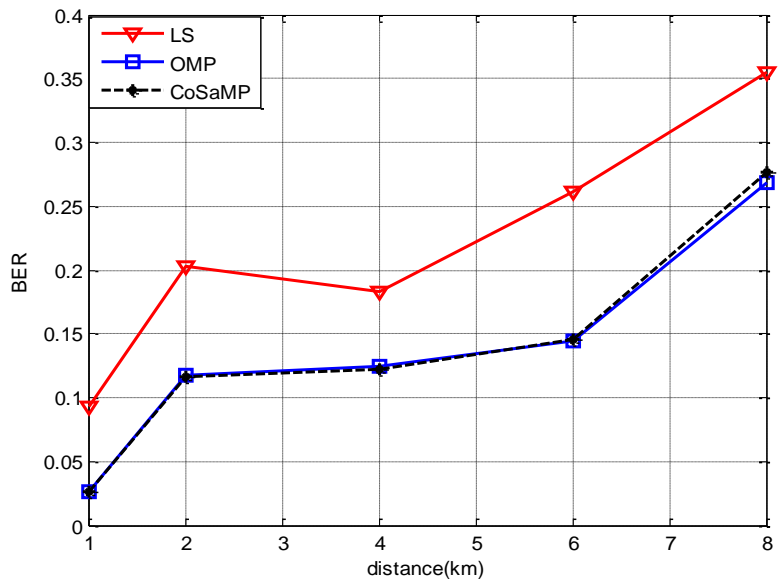

Fig. 8. BER performance of different channel estimation methods

based on the OMP algorithm and the CoSaMP algorithm perform better than the traditional LS algorithm. This is because the LS algorithm does not take advantage of the sparsity of the UA channel. The OMP algorithm and CoSaMP algorithm show similar performance in this experiment. However, considering the computational complexity, the CoSaMP algorithm is more suitable for channel estimation in a highrate UA communication system. It can also be observed from Figure 8 that as the communication distance increases, the BERs of the three algorithms increase due to the reduced SNR caused by the longer communication range.

\section{CONCLUSION}

We presented an efficient channel estimation method for high-rate UA communication that combines the advantages of block-by-block decision-directed channel tracking and the CS-based channel estimation. It was shown through our UA communication experiment that the proposed algorithm greatly increases the accuracy of time-varying UA channel estimation. Compared with the traditional LS algorithm using the same block-by-block channel tracking method, the proposed method reduced the uncoded BER by $66.7 \%$ and $46.2 \%$ in $1 \mathrm{~km}$ and 6 $\mathrm{km}$ range, respectively. The reduction of BER achieved $70 \%$ and $26.3 \%$ over that of CS-based channel estimation without the block-by-block channel tracking algorithm in $1 \mathrm{~km}$ and 6 $\mathrm{km}$ range, respectively.

\section{REFERENCES}

[1] M. Stojanovic, "Recent advances in high-speed underwater acoustic communications," IEEE J. Oceanic Engineering,vol. 21, pp. 125-136, Apr. 1996.

[2] D. B. Kilfoyle and A. B. Baggeroer, "The state of the art in underwater acoustic telemetry," IEEE J. Oceanic Engineering, vol. 25, pp. 4-27, Jan. 2000.

[3] M. Stojanovic and J. Preisig, "Underwater acoustic communication channels: Propagation models and statistical characterization," IEEE Commun. Magazine,vol. 47, pp. 84-89, Jan. 2009.

[4] R. Baraniuk, "Compressive sensing," IEEE Signal Process. Magazine, vol. 24, pp. 118-121, July 2007.

[5] E. J. Cands and M. B. Wakin, "An introduction to compressive sampling," IEEE Signal Process. Magazine,vol. 25, pp. 21-30, Mar. 2008.

[6] G. M. Davis, S. G. Mallat, and Z. Zhang, "Adaptive time-frequency decompositions with matching pursuit," Proc. SPIE SSP, 1994.

[7] J. A. Tropp and A. C. Gilbert, "Signal recovery from random measurements via orthogonal matching pursuit," IEEE Trans. Inform. Theory, vol. 53, pp. 4655-4666, Dec. 2007

[8] D. Needell and J. A. Tropp, "CoSaMP: Iterative signal recovery from noisy samples," Appl. Comput. Harmon. Anal.,vol. 26, pp. 301-321, May 2009.

[9] J. Zhang and Y. R. Zheng, "Bandwidth-efficient MIMO underwater acoustic communications with frequency-domain equalization," Proc. IEEE OCEANS, May 2010.

[10] W. U. Bajwa, J. D. Haupt, G. M. Raz, S. J. Wright, and R. D. Nowak, "Toeplitz-structured compressed sensing matrices," Proc. IEEE SSP, pp. 294-298, Aug. 2007. 